\section{Response to: 'Concerns about report suggesting glucosamine and chondroitin protect against cartilage loss' by Felson}

We wish to address the comments by Felson ${ }^{1}$ regarding our recently published article. ${ }^{2}$

This study used the osteoarthritis initiative (OAI) cohort database, which offers a unique opportunity to explore the onset and progression of knee osteoarthritis (OA) and the potential effects of treatments on the natural progression of the disease. Obviously, as does any observational study, such cohort has limitations. The rationale behind the referenced work was to build a study design that circumvented some of the limitations of the OAI cohort by selecting a subpopulation of the participants. We wanted to study, as reliably as possible, the potential diseasemodifying osteoarthritis drug (DMOAD) effects of glucosamine (Glu) and chondroitin sulfate (CS) in a design that would mimic a randomised controlled trial (RCT) with all of the restrictions that such a study design could impose in the context of an observational study. From the first-line analysis it was obvious that the patient population was heterogeneous from a clinical standpoint, with one of the main factors being related to disease treatment ( \pm analgesics/non-steroidal anti-inflammatory drugs (NSAIDs)) and the implicit severity of the disease based on symptoms and joint space width (JSW). This observation was interesting and, importantly, clinically relevant.

As outlined in the study design section, we chose to divide the cohort into two separate groups to have a better homogenisation of the studied individuals than the overall population. Clearly, participants who took analgesics/NSAIDs represented participants that were distinct from those taking only $\mathrm{Glu} / \mathrm{CS}$, likely reflecting the occurrence of symptoms. These two subsets have clear distinct baseline characteristics as demonstrated in table 1. Patients on Glu/CS and analgesics/NSAIDs were predominately women with higher body mass index (BMI), higher baseline pain and lower JSW, to name a few, which may have influenced subsequent cartilage volume loss. Therefore, we felt it was completely inappropriate to combine the two cohorts (with or without analgesics/NSAIDs), as suggested by Dr Felson, as these two subsets are clearly different clinically and combining them would not have been logical.

Moreover, in the daily practice of a clinician, there is a natural cleavage between the two patient populations identified in this study; the + analgesics/NSAIDs population being the most clinically relevant. There is, for this patient population, a desperate need to answer the question as to whether or not treatment with agents such as Glu/CS can offer a potential protective effect on the progression of OA. Consequently, there was clearly a clinically relevant and practical logic to the selection of patient cohorts for this study.

In the letter, the assumption that there is no association between Glu/CS and protection against cartilage volume loss in patients taking analgesics/NSAIDs has to be tempered. Indeed, in table 3, the majority of the subregions showed a higher cartilage volume loss over time in patients not taking Glu/CS compared with those taking Glu/CS, and significance was reached for some, as described in the Results section. Unfortunately, when adding covariates it was difficult to demonstrate a difference in the model, due to lack of statistical power. Still, multivariate analysis (presented in the text of the Results section) using BMI and the Western Ontario and McMaster Universities Osteoarthritis Index (WOMAC) as covariates demonstrated a numerical trend $(p=0.07)$ for the global plateau in favour of the subgroup taking Glu/CS, which we feel is most relevant. We strongly believe that this merely reflects the lack of statistical power as opposed to the absence of a therapeutic effect. The fairly low number of eligible subjects was also a reason why we did not match patients on analgesics/NSAIDs versus those not treated, as suggested by Dr Felson, in order to provide balanced groups at baseline and optimally assess cartilage loss over time.

In regard to the question of the effect of $\mathrm{Glu} / \mathrm{CS}$ on the medial versus lateral compartment, as explained at length in the Discussion section, the effect probably relates to the severity of the disease. To answer the clinical relevance of protecting the lateral compartment, the finding of a preferential reduction in cartilage loss by a drug in this compartment is consistent with the results of a number of RCTs using MRI. ${ }^{3-6}$ Although the exact reasons for this preferential protective effect remain unknown, one possible explanation could be that in the lateral compartment where cartilage lesions are less severe, the capacity of the tissue to repair under treatment may be greater. The role of abnormal biomechanical forces and/or malalignment could also be factors contributing to those changes. Finally, the clinical relevance of the protective effect of treatment on the lateral compartment has been demonstrated in two of the previously mentioned studies, ${ }^{45}$ in which a reduction in cartilage volume in this compartment was demonstrated to correlate with a reduction in the need for total knee replacement. ${ }^{78}$

Regarding the conclusion of the letter, we would like to highlight the fact that the present findings do not stand alone but rather complement and substantiate multiple studies $\left(\mathrm{Glu}^{9}{ }^{10}\right.$ and $\mathrm{CS}^{3}{ }^{11-13}$ ), indicating that Glu and CS do have a protective effect on cartilage loss in patients with knee OA and corroborate with studies showing that treatment with $\mathrm{Glu}^{14}$ and $\mathrm{CS}^{8}{ }^{15}$ has a positive impact on the disease outcome.

In summary, the data of the referenced study should be analysed in the context of all of the elements stated above. Dr Felson's letter does not render justice to the data presented or the complexity involved in the analyses of the OAI cohort. As OA is a very heterogeneous disease, implying that patients will not uniformly respond to a given therapy, it is therefore critical to identify patient subgroups that are likely to benefit from a targeted therapy.

Johanne Martel-Pelletier, ${ }^{1}$ Camille Roubille, ${ }^{1}$ François Abram, ${ }^{2}$ Marc Hochberg, ${ }^{3}$ Marc Dorais, ${ }^{4}$ Philippe Delorme, ${ }^{5}$ Jean-Pierre Raynauld, ${ }^{1}$ Jean-Pierre Pelletier ${ }^{1}$

${ }^{1}$ Osteoarthritis Research Unit, University of Montreal Hospital Research Centre (CRCHUM), Montreal, Quebec, Canada

${ }^{2}$ Medical Imaging Research \& Development, ArthroLab Inc., Montreal, Quebec, Canada

${ }^{3}$ Departments of Medicine and Epidemiology and Public Health, University of Maryland School of Medicine, Baltimore, Maryland, USA

${ }^{4}$ StatSciences Inc., Notre-Dame de l'Île-Perrot, Quebec, Canada

${ }^{5}$ ArthroLab Inc., Montreal, Quebec, Canada

Correspondence to Professor Johanne Martel-Pelletier, Osteoarthritis Research Unit, University of Montreal Hospital Research Centre (CRCHUM), Montreal, QC, Canada H2X 0A9; jm@martelpelletier.ca

Contributors All authors have read and approved this letter.

Competing interests JM-P, MH and J-PP are consultants for Bioiberica, S.A. MH is principal investigator of the Baltimore Clinical Center of the OAI (University of Maryland). JM-P, CR, J-PR, FA and J-PP, M Dorais and PD are not part of the OAI investigative team. JM-P and J-PP are shareholders in ArthroLab Inc. J-PR and M Dorais are consultants for ArthroLab Inc. FA and PD are employees of ArthroLab Inc.

Provenance and peer review Commissioned; internally peer reviewed.

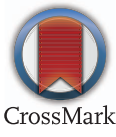


To cite Martel-Pelletier J, Roubille C, Abram F, et al. Ann Rheum Dis 2015;74:e39.

Received 9 March 2015

Accepted 15 March 2015

Published Online First 1 April 2015

\section{SLinked}

http://dx.doi.org/10.1136/annrheumdis-2015-207479

Ann Rheum Dis 2015;74:e39. doi:10.1136/annrheumdis-2015-207491

\section{REFERENCES}

1 Felson DT. Concerns about report suggesting glucosamine and chondroitin protect against cartilage loss. Ann Rheum Dis 2015;74:e38.

2 Martel-Pelletier J, Roubille C, Abram F, et al. First-line analysis of the effects of treatment on progression of structural changes in knee osteoarthritis over 24 months: data from the osteoarthritis initiative progression cohort. Ann Rheum Dis 2015:74:547-56

3 Wildi LM, Raynauld JP, Martel-Pelletier J, et al. Chondroitin sulphate reduces both cartilage volume loss and bone marrow lesions in knee osteoarthritis patients starting as early as 6 months after initiation of therapy: a randomised, double-blind, placebo-controlled pilot study using MRI. Ann Rheum Dis 2011;70:982-9.

4 Raynauld JP, Martel-Pelletier J, Bias P, et al. Protective effects of licofelone, a 5-lipoxygenase and cyclo-oxygenase inhibitor, versus naproxen on cartilage loss in knee osteoarthritis: a first multicentre clinical trial using quantitative MRI. Ann Rheum Dis 2009;68:938-47.

5 Pelletier JP, Kapoor M, Fahmi H, et al. Strontium ranelate reduces the progression of experimental dog osteoarthritis by inhibiting the expression of key proteases in cartilage and of IL-1beta in the synovium. Ann Rheum Dis 2013;72:250-7.
6 Lohmander LS, Hellot S, Dreher D, et al. Intraarticular sprifermin (recombinant human fibroblast growth factor 18) in knee osteoarthritis: a randomized, double-blind, placebo-controlled trial. Arthritis Rheumatol 2014;66: 1820-31.

7 Raynauld JP, Martel-Pelletier J, Haraoui B, et al. Risk factors predictive of joint replacement in a 2-year multicentre clinical trial in knee osteoarthritis using MRI: results from over 6 years of observation. Ann Rheum Dis 2011;70:1382-8.

8 Raynauld JP, Martel-Pelletier J, Dorais M, et al. Total knee replacement as a knee osteoarthritis outcome: predictors derived from a 4 year long-term observation following a randomized clinical trial using chondroitin sulfate. Cartilage 2013:4:219-26.

9 Pavelka K, Gatterova J, Olejarova M, et al. Glucosamine sulfate use and delay of progression of knee osteoarthritis: a 3-year, randomized, placebo-controlled, double-blind study. Arch Intern Med 2002;162:2113-23.

10 Reginster JY, Deroisy R, Rovati LC, et al. Long-term effects of glucosamine sulphate on osteoarthritis progression: A randomised, placebo-controlled clinical trial. Lancet 2001;357:251-6.

11 Kahan A, Uebelhart D, De Vathaire F, et al. Long-term effects of chondroitins 4 and 6 sulfate on knee osteoarthritis: The study on osteoarthritis progression prevention, a two-year, randomized, double-blind, placebo-controlled trial. Arthritis Rheum 2009;60:524-33

12 Bruyere 0 , Reginster JY. Glucosamine and chondroitin sulfate as therapeutic agents for knee and hip osteoarthritis. Drugs Aging 2007;24:573-80.

13 Michel BA, Stucki G, Frey D, et al. Chondroitins 4 and 6 sulfate in osteoarthritis of the knee: A randomized, controlled trial. Arthritis Rheum 2005;52:779-86.

14 Bruyere O, Pavelka K, Rovati LC, et al. Total joint replacement after glucosamine sulphate treatment in knee osteoarthritis: results of a mean 8-year observation of patients from two previous 3-year, randomised, placebo-controlled trials. Osteoarthritis Cartilage 2008;16:254-60.

15 Hochberg MC. Structure-modifying effects of chondroitin sulfate in knee osteoarthritis: an updated meta-analysis of randomized placebo-controlled trials of 2-year duration. Osteoarthritis Cartilage 2010;18(Suppl 1):S28-31. 